\title{
Buffer Management in Mobile Social Networks
}

\author{
Ahmed B. Altamimi* \\ University Oh Hail/Department of Computer Science and Software Engineering, Hail, Saudi Arabia. \\ * Corresponding author. Tel: 00966920005995; email: Altamimi.a@gmail.com \\ Manuscript submitted September 24, 2015; accepted December 30, 2015. \\ doi: $10.17706 /$ jcp.12.1.20-27
}

\begin{abstract}
A mobile social network (MSN) is defined as a mobile network that uses social relationships to determine node communication. There has been extensive research on routing protocols for MSNs. These protocols assume cooperating nodes carry messages to the destination nodes. Node cooperation may be limited due to buffer concerns. Thus, buffer management strategy is proposed here for MSN routing protocols. Performance results are presented which show that this approach leads to improve the probability of delivery.
\end{abstract}

Key words: Buffer management technique, delivery probability, mobile social network, node cooperation.

\section{Introduction}

A wireless network allows mobile users to communicate without a physical connection such as a cable. Wireless networks now offer high data rates and reliability at low cost. This has led to a rapid and widespread increase in their use in recent years. A wireless network can be organized in three ways. First, a fixed network infrastructure with access points can be used. With this approach, mobile nodes communicate with other nodes through access points. The drawback of this system is that when a node moves from one access point to another, delay and packet loss can occur, and coverage is an issue. The second approach is to form an ad hoc network to allow nodes to communicate. In this case, each node has the ability to route a message to the destination without the existence of a fixed infrastructure. Nodes can track each other by sending control messages when they move, providing the information required to forward a message to its destination. However, the resources required to monitor and maintain routes to other nodes can be significant. In addition, an ad hoc network is limited in size by the transmission range of a node. This size is typically much smaller than the range available using access points. To overcome these limitations, an intermittently connected network (ICN) can be considered In this case, nodes can route messages to their destinations without keeping track of the movement of other nodes.

Intermittently connected networks (ICNs) have been the subject of much research because they assume node mobility without the existence of a permanent connection between nodes. Although this assumption offers great flexibility, it creates routing challenges. In fact, existing routing protocols for ad hoc networks are not applicable in this case because routes to most nodes are unlikely to exist. Thus, routing methods have been proposed for ICNs which assume that a path between a source and destination is nonexistent. These can be classified based on the choice of the next carrier of a message as opportunistic forwarding, prediction based, or social relationship based.

With opportunistic forwarding, nodes in the network forward their messages to any encountered node. In the case of prediction based methods, only nodes with a higher probability of delivering a message to the 
destination based on their contact history are selected. Finally, social relationship based methods use the social relationship with the message destination to determine which nodes to forward a message to. Many ICN protocols have been proposed in the literature. For example, opportunistic forwarding was employed in [1] and [2], prediction based forwarding was used in [3], and social relationship based forwarding was used in [4]. Because of the rapid increase in the number of social network users [5], many techniques have focused on social relationship based forwarding. A network which uses this approach is defined as a mobile social network (MSN).

The goal of most protocols (including MSN protocols), is to achieve a high probability of delivery. This probability is defined as the ratio of the number of messages received by destination nodes to the number of messages sent by source nodes. To achieve a high delivery probability, node cooperation is required. However, node cooperation is subject to buffer availability. Existing MSN routing protocols assume all nodes cooperate, but in a practical network some nodes may be unwilling to participate because this requires that nodes free its buffer when it is full. The question become which message should be discarded to accept a new encountered message. Thus, a buffer management technique for mobile social network routing protocols is developed here.

\section{Related Work}

Mobile Social Network (MSN) Routing Protocols: A mobile social network (MSN) is defined as a mobile network that uses social relationships or activities to coordinate node communication. Routing in these networks uses either similar social interests (activities) or similar geographic environments. For example, LABEL [6], SocialCast [7], and STATUS [4] use the interest similarities between an encountered node and the destination to determine if a message is forwarded. SimBet [8] uses the similarity of the geographic environment of an encountered node to forward a message. In particular, forwarding is based on the number of neighbours of a node. Bubble [9] uses a combination of these techniques to route messages. The goal of MSN routing protocols is to provide a high delivery probability with low complexity.

Buffer Management in Mobile Social Network (MSN): Some work have been conducted to address buffer management in delay tolerant networks [10], [11]. These works mainly propose a buffer management technique for routing protocol in DTN, whereas the focus of this work is buffer management in MSN.

\section{Buffer Management in MSNs}

Buffer management is a key concern in MSNs. This is because a cooperating node must have a buffer management technique that allow them to cooperate. Node cooperation is essential in MSNs to obtain a high delivery probability [12]-[14], but to ensure this, a buffer management technique must be provided.

The STATUS MSN protocol [4] is considered in this paper. With this protocol, when a node is encountered a message is forwarded based on two factors. First, if the encountered node has a status, it may receive a copy of the message. Having a status means that the encountered node is going to a point of interest (PoI). A PoI is expected to have many nodes located there, such as a shopping mall or a park. Second, a message is forwarded to an encountered node if this node lives in the neighborhood of the destination node.

The performance of Status, just like any other ICN routing protocol, is expected to increase in terms of delivery ratio if a suitable buffer management technique is provided.

One approach to improving buffer management is to form groups of similar communities. For example, shopping malls can be considered as a group as they can be expected to have a similar number of visitors and are similar in size.

Therefore, a node exchange its buffered messages with an encountered node based on the destination 
class of the encountered node. For example, if a node is going to a restaurant with a large capacity (e.g. over 500 people a day), it can state that it is going to restaurant class $\mathrm{A}$, which only denotes that the expected number of visitor is over 500. In this case, a copy of a message is given to a node that is going to a PoI that is expected to have large number of visitors. This leads not to consume every encountered node's buffer.

Fig. 1 illustrates the buffer management method proposed for the STATUS protocol. The top diagram shows nodes with the standard STATUS routing protocol, whereas the bottom diagram shows nodes with buffer management technique. In this case, a message is forwarded to an encountered node based on its class. For example, if a message exchanged only to the top $25 \%$ of busiest locations, this means that an encountered node will receive a copy of a message if it is headed to class A, which is expected to have 1000 visitors. Whereas, if a message exchanged with top $75 \%$ locations, a message is forwarded to class $\mathrm{A}, \mathrm{B}$, and $\mathrm{C}$ since they consider the top 3 out of 4 busiest locations in terms of visitors. The percentage of visitors is used to determine the class of a location. Note that the proposed approach to buffer management can be adopted with any MSN routing protocol.

Although high nodes cooperation to deliver a message is desired in MSNs, it might not be the case if 1) the messages are forwarded to locations that are not expected to have many visitors or/and 2)the buffer size is limited. This will reduce their chances to be delivered to the final destinations, in particular, when a number of message copies are predetermined. With predetermined number of message copies, the copies should be delivered to the busiest location in MSN, so their probability of meeting the destination is increased. Additionally, with limited buffer size, it would be desired not to consume an encountered node' buffer, unless if it is expected to travel to busy location.
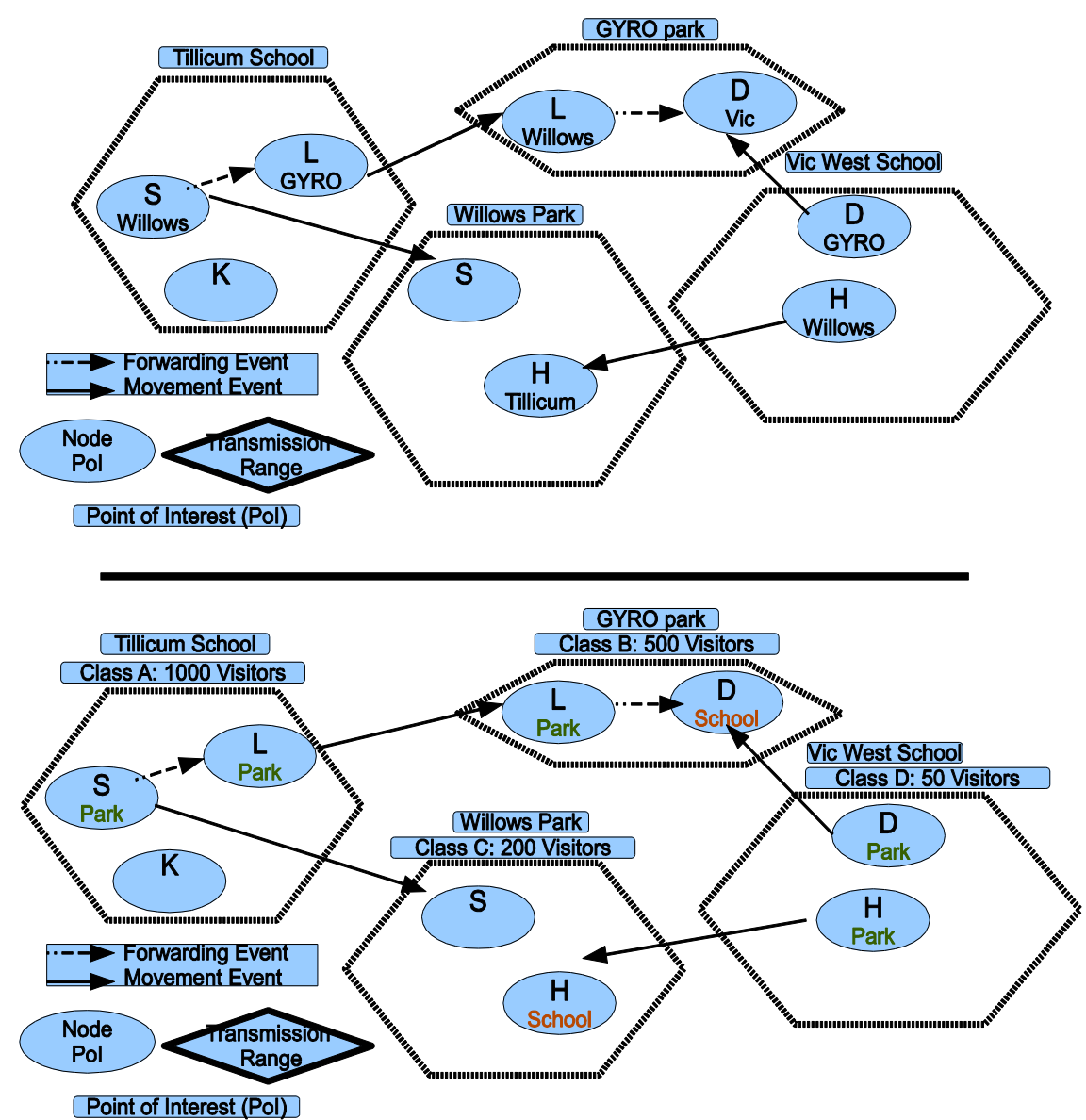

Fig. 1. An illustration of forwarding process with the STATUS protocol when buffer management is employed. 


\section{Performance Evaluation}

\subsection{The ONE Simulator}

ONE [15] is a discrete event simulation package. It combines movement modeling, routing, visualization and reporting. Mobility models determine node movement within the simulation environment. The random waypoint model (RWPM) is widely used and is based on random directions and speeds. However, this random node movement is unrealistic when mobile devices are carried by humans. It is more pragmatic to assume that nodes move towards a specific destination, then another destination, and so on. These destinations are typically particular locations such as malls, restaurants or schools, and so are called points of interest (PoI). The more realistic shortest path movement model (SPMM) has nodes moving towards particular locations, and so is employed here.

\subsection{Simulation Environment}

The simulation parameters employed are from the realistic environment described in [4]. There are 120 nodes moving towards 30 PoIs and 30 neighborhoods in an area of size $4500 \times 3400 \mathrm{~m}^{2}$. Nodes are randomly assigned to neighborhoods. A node returns to its neighborhood after each pair of visits to PoIs. A percentage of the nodes are assumed to visit each PoI. This percentage is defined with respect to the other PoIs locations. For example, a PoI has a $10 \%$ visit percentage if it is visited $90 \%$ less frequently than the location visited most frequently. The 30 location percentages are uniformly distributed from the set $\{10 \%$, $20 \%, \ldots, 100 \%$. Each node represents a user moving with a realistic speed along the shortest path between locations randomly chosen based on their visit percentages. The simulation environment parameters are summarized in Table 1.

Table 1. Simulation Environment Parameters

\begin{tabular}{ccccc}
\hline \hline Parameter & Transmit Rate & Transmit Range & Node Speed & Message Size \\
\hline Value & $250 \mathrm{KBps}$ & $50 \mathrm{~m}$ & $.5-2.5 \mathrm{~m} / \mathrm{s}$ & $.5-4 \mathrm{MB}$ \\
& & & & \\
\hline \hline
\end{tabular}

\subsection{Performance Results}

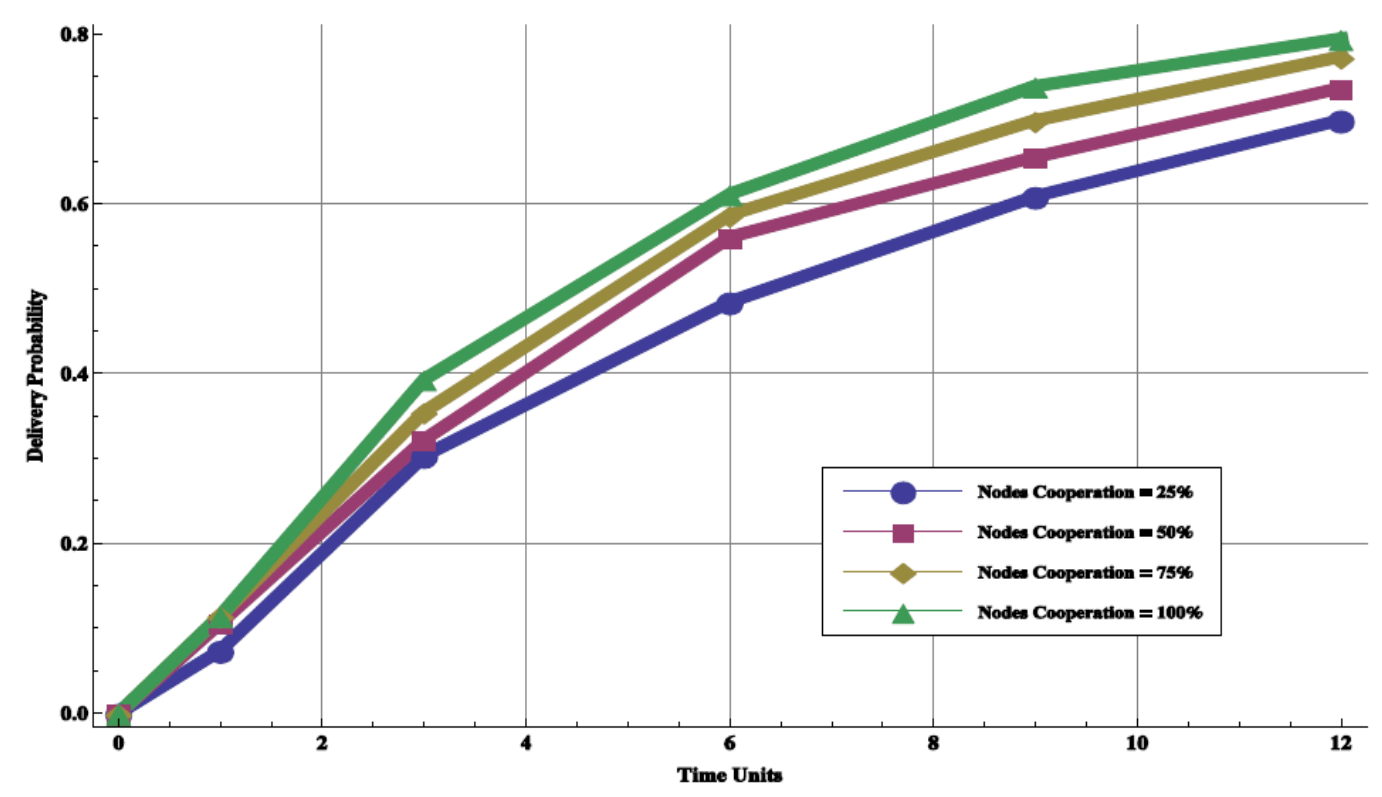

Fig. 2. The message delivery probability for the STATUS protocol with large buffer size and varying levels of node cooperation. 
STATUS [4] is a mobile social network (MSN) routing protocol. It was shown in [4] that STATUS outperforms other MSN routing protocols such as epidemic [1], spray and wait [2], and LABEL [6]. Thus, STATUS is employed here as a suitable MSN routing protocol. The delivery probability of STATUS is considered with and without privacy implemented, and the impact of the percentage of cooperating nodes is examined. Each message is assumed to have $L=10$ copies that can be distributed.

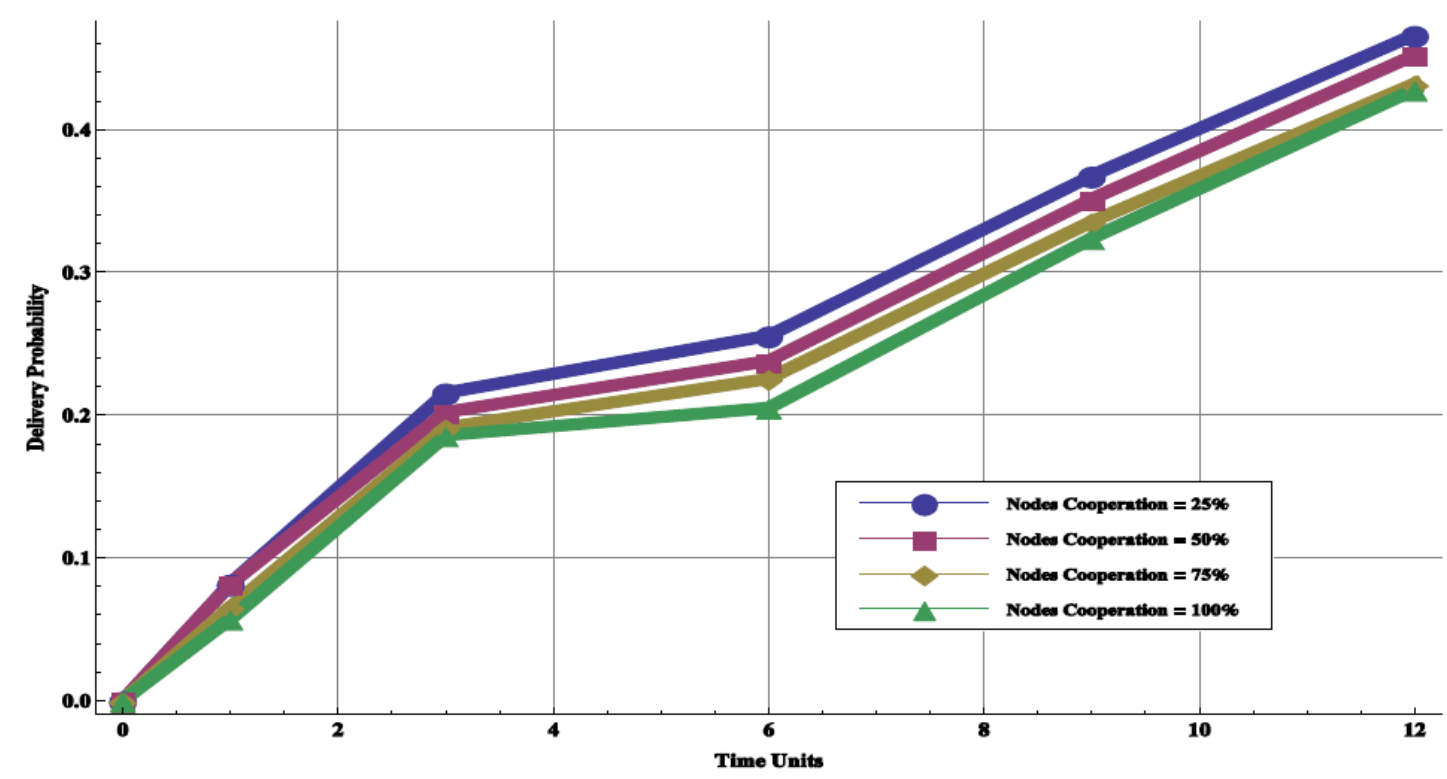

Fig. 3. The message delivery probability for the STATUS protocol with limited buffer size and varying levels of node cooperation.

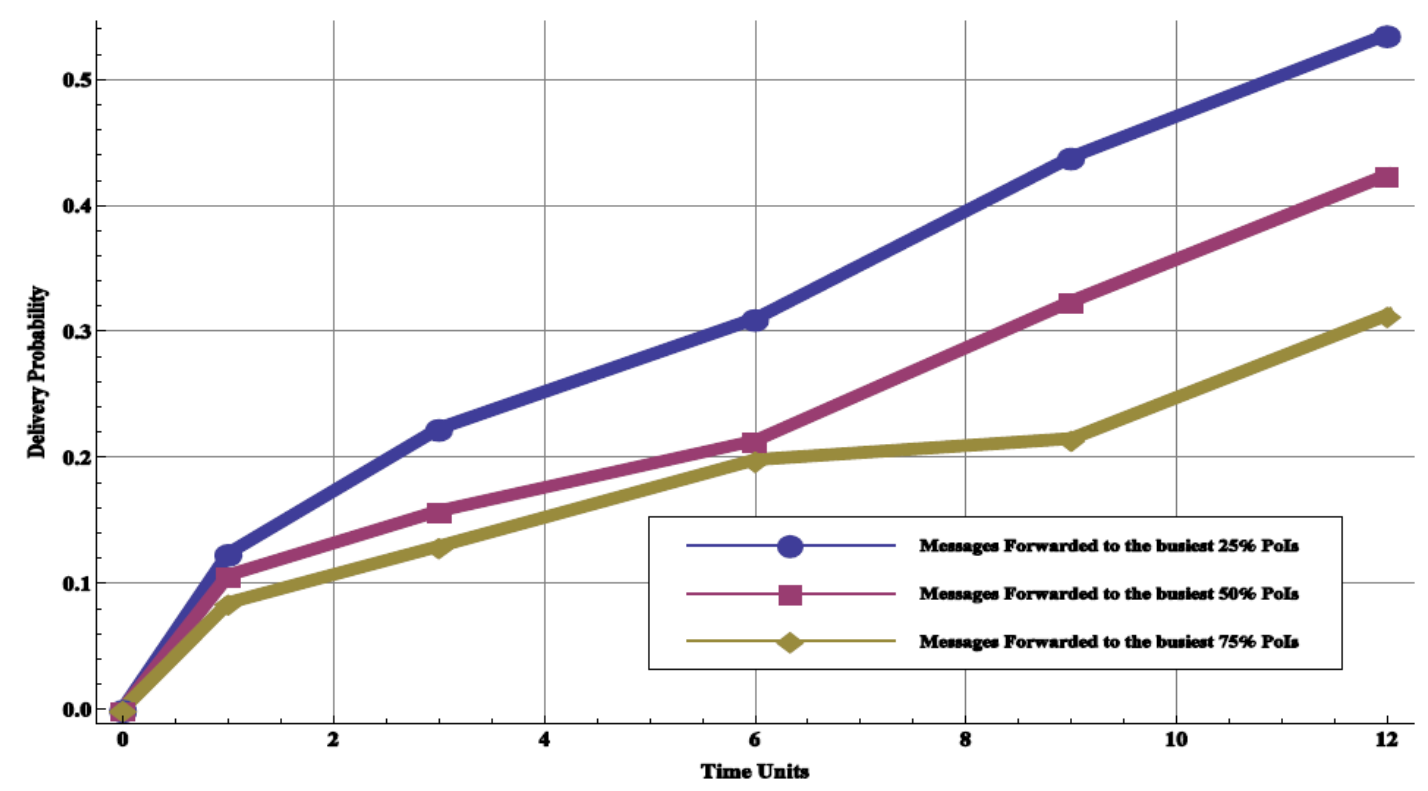

Fig. 4. The message delivery probability for the STATUS protocol with $5 \mathrm{M}$ buffer size, varying levels of nodes that messages are exchanged with and $100 \%$ nodes cooperation.

Figs. 2 and 3 shows the delivery probability for the STATUS when large and small buffer size is employed, respectively, with vary nodes of cooperation percentage. This probability is defined as the ratio of the number of messages received by destination nodes to the number of messages sent by source nodes. Fig. 2 indicates that STATUS has a better delivery probability when the cooperated nodes increased with large buffer size. However, Fig. 3 shows that the delivery probability does not always increase when the 
percentage of cooperated nodes increase, in particular, when a small buffer size is employed. This is because the small buffer size does not necessary need a large percentage of nodes cooperation, but it needs to exchange messages to cooperated node that help to deliver messages faster before any messages' drop occur due to buffer overflow. Thus, increasing nodes cooperation does not always increase the delivery probability without a proper buffer management technique.

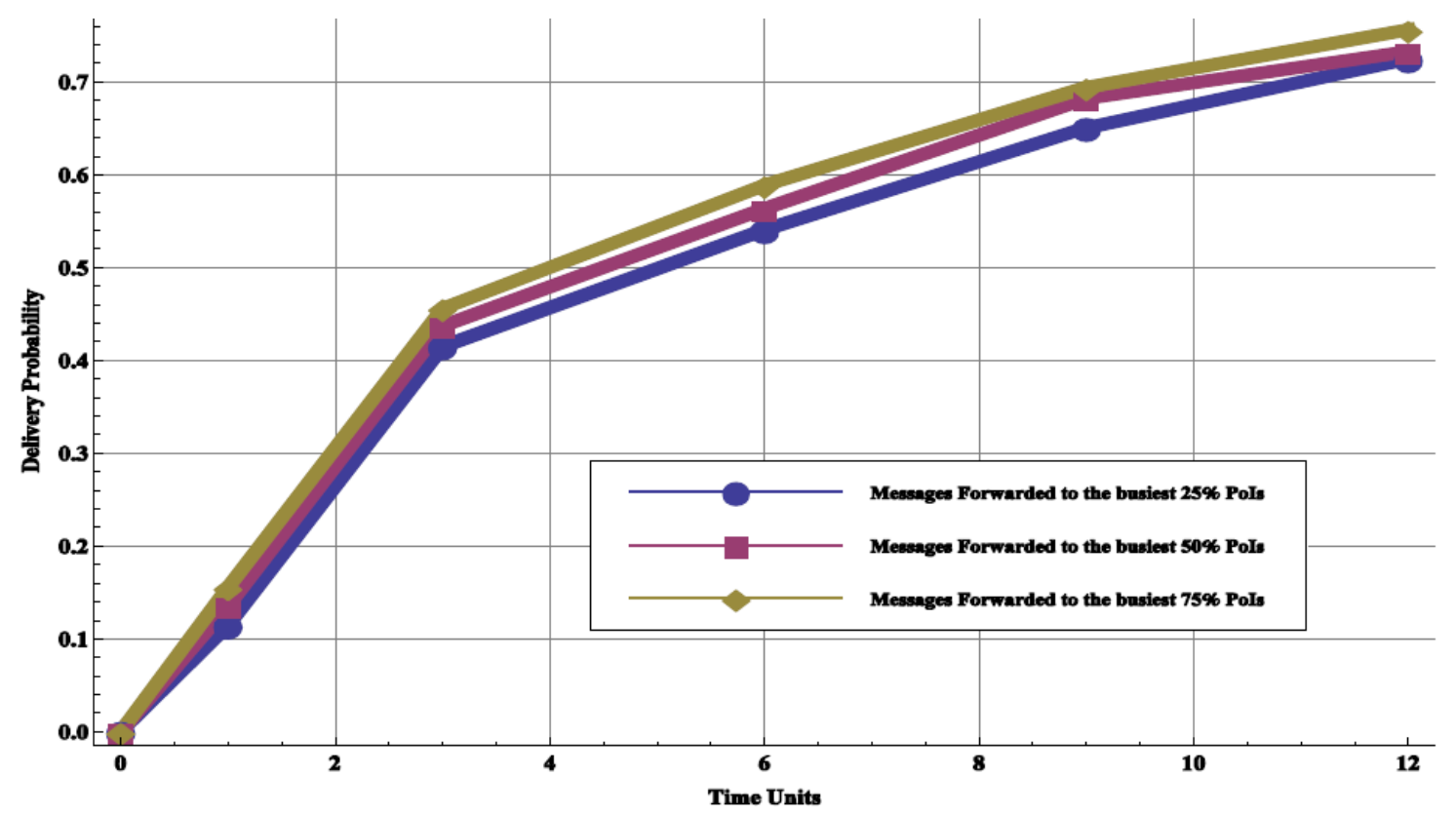

Fig. 5. The message delivery probability for the STATUS protocol with 100M buffer size, varying levels of nodes that messages are exchanged with and $100 \%$ nodes cooperation.

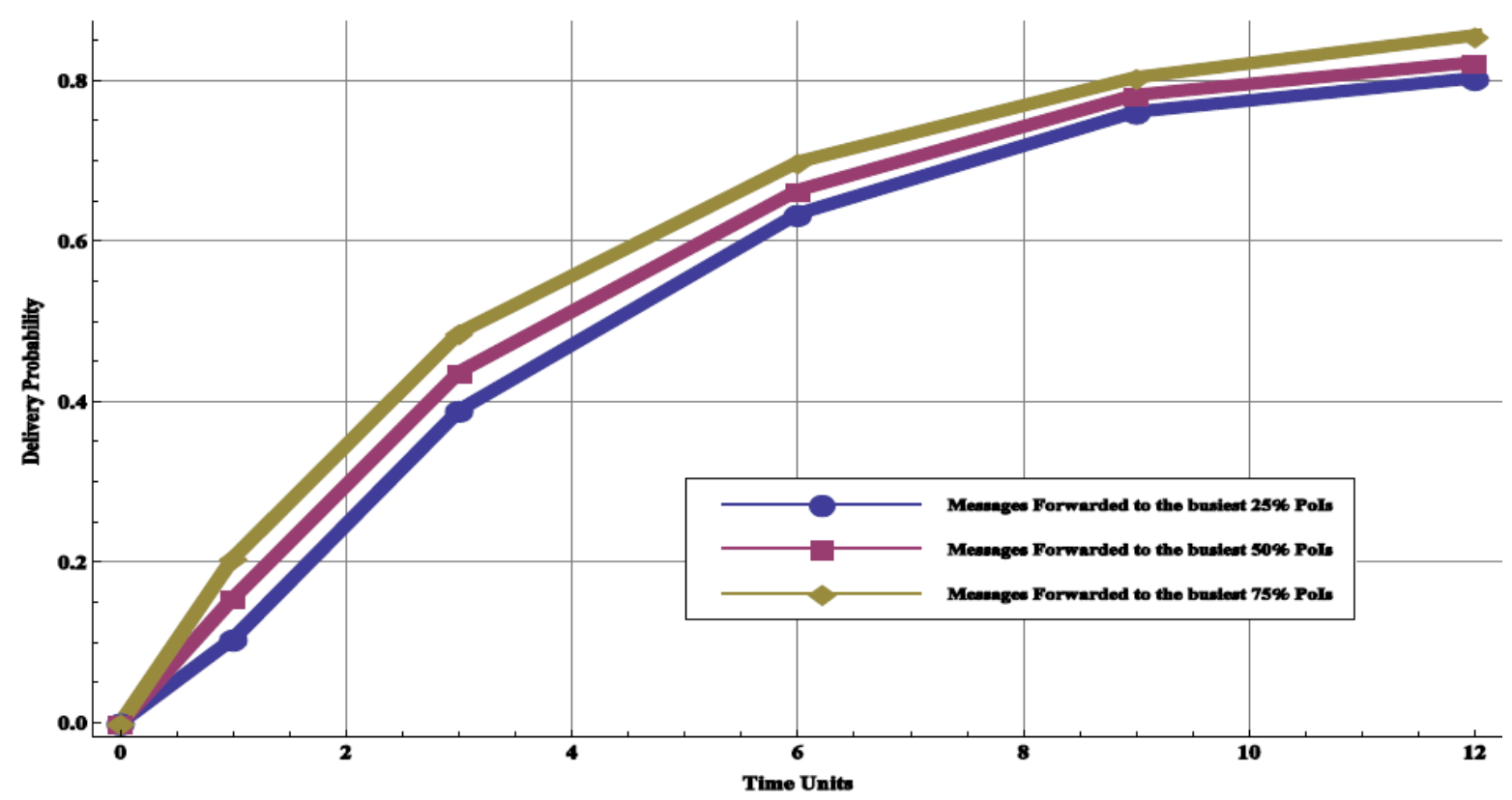

Fig. 6. The message delivery probability for the STATUS protocol with $2 \mathrm{G}$ buffer size, varying levels of nodes that messages are exchanged with and $100 \%$ nodes cooperation.

Figs. 4, 5 and 6 shows the delivery probability for the STATUS when buffer management technique is employed with $5 \mathrm{M}, 100 \mathrm{M}$ and $2 \mathrm{G}$ buffer size, respectively. The figures shows the delivery probability when messages forwarded to the busiest locations with vary percentage. It was shown in Fig. 3 that increasing 
the nodes cooperation decreases the delivery probability when a small buffer size is employed. However, in Fig. 4 , with the same limited buffer size, the delivery probability is increased with node cooperation $100 \%$ when the protocol limit messages exchange to the encountered nodes that are heading to the busiest locations.

The impact of the buffer management technique is reduced when large buffer is employed, as shown in Figs. 5 and 6. These figures shows that increasing nodes cooperation leads to improve the delivery probability, regardless of the employed buffer management technique.

\section{Conclusion}

A buffer management technique was presented to improve the performance of mobile social network (MSN) routing protocols. In particular, the MSN routing protocol STATUS was enhanced by introducing buffer management. Performance results were presented which show that ensuring node buffer management can improve the delivery probability. This is a result of forwarding messages copies to the busiest locations in MSNs. As expected with large buffer size, the greater the node cooperation, the higher the delivery probability. With limited buffer size, the greater the node cooperation, the higher the delivery probability when buffer management technique is employed.

\section{References}

[1] Vahdat, A., \& Becker, D. (2000). Epidemic Routing for Partially Connected Ad Hoc Networks (Tech. Rep. CS-200006, 2000). Durham, NC, USA.: Duke University.

[2] Spyropoulos, T., Psounis, K., \& Raghavendra, C. (2005). Spray and wait: An efficient routing scheme for intermittently connected mobile networks. Proceedings of ACM SIGCOMM Workshop on Delay Tolerant Networking (pp. 252-259). Philadelphia, PA.

[3] Lindgren, A., Doria, A., \& Schelen, O. (2003). Probabilistic routing in intermittently connected networks. ACM SIGMOBILE Mobile Computing and Commun. Review, 7(3), 19-20.

[4] Altamimi, A. B., \& Gulliver, T. A. (2012). On routing protocols using mobile social networks. Int. J. Wireless and Mobile Comput., 7(3), 1-11.

[5] Social networking statistics, Statistics Brain. (Aug. 2013). [Online]. Available: http://www.statisticbrain.com/social-networking-statistics/

[6] Hui, P., \& Crowcroft, J. (2007). How small LABELS create big improvements. Proceedings of IEEE Int. Conf. on Pervasive Computing and Commun. Workshops (pp. 65-70). White Plains, NY.

[7] Costa, P., Mascolo, C., Musolesi, M., \& Picco, G. P. (2008). Socially-aware routing for publish-subscribe in delay-tolerant mobile ad hoc networks. IEEE J. Select. Areas Commun., 26(5), 748-760.

[8] Daly, E. M., \& Haahr, M. (2007). Social network analysis for routing in disconnected delay-tolerant MANETs. Proceedings of ACM Int. Symp. On Mobile Ad Hoc Networking and Computing (pp. 32-40). Montreal, QC.

[9] Hui, P., Crowcroft, J., \& Yoneki, E. (2008). Bubble rap: Social based forwarding in delay tolerant networks. Proceedings of ACM Int. Symp. on Mobile Ad hoc Networking and Computing (pp. 1576-1589). Hong Kong.

[10] Lindgren, A., \& Phanse, K. S. (2006). Evaluation of queuing policies and forwarding strategies for routing in intermittently connected networks. Proceedings of IEEE COMSWARE. New Delhi.

[11] Krifa, A., Barakat, C., \& Spyropoulos, T. (2008). Optimal buffer management policies for delay tolerant networks. Proceedings of 5th Annual IEEE Communications Society Conference on Sensor, Mesh and Ad Hoc Communications and Networks (pp. 260-268). San Francisco, CA.

[12] Panagakis, A., Vaios, A., \& Stavrakakis, I. (2007). On the effects of cooperation in DTNs. Proceedings of 
Int. Conf. on Commun. Systems, Software and Middleware (pp. 1-6). Bangalore, India.

[13] Resta, G., \& Santi, P. (2009). The effects of node cooperation level routing performance in delay tolerant networks. Proceedings of IEEE Conf. on Sensor, Mesh and Ad Hoc Commun. and Networks (pp. 1-9). Rome, Italy.

[14] Keranen, A., Pitk"anen, M., Vuori, M., \& Ott, J. (2011). Effect of non cooperative nodes in mobile DTNs. Proceedings of IEEE WoWMoM Workshop on Autonomic and Opportunistic Commun (pp. 1-7). Lucca, Italy.

[15] Ker"anen, A., Ott, J., \& K"arkk"ainen, T. (2009). The ONE simulator for DTN protocol evaluation. Proceedings of Int. Conf. on Simulation Tools and Techniques (pp. 1-10). Rome, Italy.

[16] Dijkstra, E. W. (1959). A note on two problems in connexion with graphs. Num. Math., 1(1), 269-271.

Ahmed B. Altamimi received the M.A.Sc. degree in electrical and computer engineering from the University of Victoria, Victoria, BC, Canada in 2010, and the Ph.D. degree in electrical and computer engineering from the University of Victoria in 2014. His research interests include routing and mobility modeling in wireless networks. 